\title{
Comparison of the MSMS and NanoShaper molecular surface triangulation codes in the TABI Poisson-Boltzmann solver
}

\author{
Leighton Wilson, Robert Krasny ${ }^{\dagger}$
}

October 22, 2020

\begin{abstract}
The Poisson-Boltzmann (PB) implicit solvent model is a popular framework for studying the electrostatics of biomolecules immersed in water with dissolved salt. In this model the dielectric interface between the biomolecule and solvent is often taken to be the molecular surface or solvent-excluded surface (SES), and the quality of the SES triangulation is critical in boundary element simulations of the PB model. In this work we compare the MSMS and NanoShaper surface triangulation codes for a set of 38 biomolecules. While MSMS produces triangles of exceedingly small area and large aspect ratio, the two codes yield comparable values for the SES surface area and electrostatic solvation energy, where the latter calculations were performed using the treecode-accelerated boundary integral (TABI) PB solver. However we found that Nanoshaper is more efficient and reliable than MSMS, especially when parameters are set to produce highly resolved triangulations.
\end{abstract}

Keywords: $\quad$ solvated biomolecule, solvent excluded surface, electrostatics, Poisson-Boltzmann, boundary element method, treecode

*Department of Mathematics, University of Michigan, Ann Arbor, MI 48109

${ }^{\dagger}$ Department of Mathematics, University of Michigan, Ann Arbor, MI 48109 


\section{INTRODUCTION}

Implicit solvent models play a key role in computational modeling of electrostatic interactions between biomolecules and their solvent environment ${ }^{1-3}$. Of particular importance is the Poisson-Boltzmann (PB) implicit solvent model ${ }^{45}$. Figure 1 shows the interior domain $\Omega_{1} \subset \mathbb{R}^{3}$ containing the solute biomolecule, the exterior domain $\Omega_{2}=\mathbb{R}^{3} \backslash \bar{\Omega}_{1}$ containing the ionic solvent, and the dielectric interface $\Gamma=\bar{\Omega}_{1} \cap \bar{\Omega}_{2}$. In a 1:1 electrolyte at low ionic concentration, one can utilize the linearized PB equation for the electrostatic potential $\phi$,

$$
-\nabla \cdot(\varepsilon(\mathbf{x}) \nabla \phi(\mathbf{x}))+\bar{\kappa}^{2}(\mathbf{x}) \phi(\mathbf{x})=\sum_{k=1}^{N_{c}} q_{k} \delta\left(\mathbf{x}-\mathbf{y}_{k}\right), \quad \mathbf{x} \in \mathbb{R}^{3},
$$

where $\varepsilon(\mathbf{x})$ is the dielectric constant, $\bar{\kappa}$ is the modified Debye-Hückel inverse length in units of $\AA^{-1}, N_{c}$ is the number of atoms in the solute biomolecule, $\mathbf{y}_{k}$ is the position of the $k$ th solute atom, and $q_{k}$ is the associated partial charge in units of fundamental charge $e_{c}$. The dielectric interface conditions are

$$
\phi_{1}(\mathbf{x})=\phi_{2}(\mathbf{x}), \quad \varepsilon_{1} \frac{\partial \phi_{1}(\mathbf{x})}{\partial n}=\varepsilon_{2} \frac{\partial \phi_{2}(\mathbf{x})}{\partial n}, \quad \mathbf{x} \in \Gamma,
$$

where $\phi_{1}(\mathbf{x})$ and $\phi_{2}(\mathbf{x})$ are the limiting values approaching the interface $\Gamma$ from inside and outside the biomolecule, respectively, and $n$ indicates the outward normal direction on the interface. The first condition in Eq. (2) expresses continuity of the potential across the interface, and the second condition expresses continuity of the electric flux. The far-field boundary condition is

$$
\lim _{|\mathbf{x}| \rightarrow \infty} \phi(\mathbf{x})=0 .
$$

The present work assumes that $\varepsilon$ and $\bar{\kappa}$ are piecewise constant,

$$
\varepsilon(\mathbf{x})=\left\{\begin{array}{ll}
\varepsilon_{1}, & \mathbf{x} \in \Omega_{1}, \\
\varepsilon_{2}, & \mathbf{x} \in \Omega_{2},
\end{array} \quad \bar{\kappa}^{2}(\mathbf{x})= \begin{cases}0, & \mathbf{x} \in \Omega_{1}, \\
\left(\frac{8 \pi N_{A} e_{c}^{2}}{1000 k_{B} T}\right) I_{s}, & \mathbf{x} \in \Omega_{2},\end{cases}\right.
$$

where $N_{A}$ is Avogadro's number, $k_{B}$ is the Boltzmann constant, $T$ is the temperature, and $I_{s}$ is the molar concentration of the ionic solvent. A key quantity of interest in analyzing protein stability is the electrostatic solvation energy,

$$
E_{\mathrm{sol}}=\frac{1}{2} \sum_{k=1}^{N_{c}} q_{k} \phi_{\mathrm{reac}}\left(\mathbf{y}_{k}\right)
$$




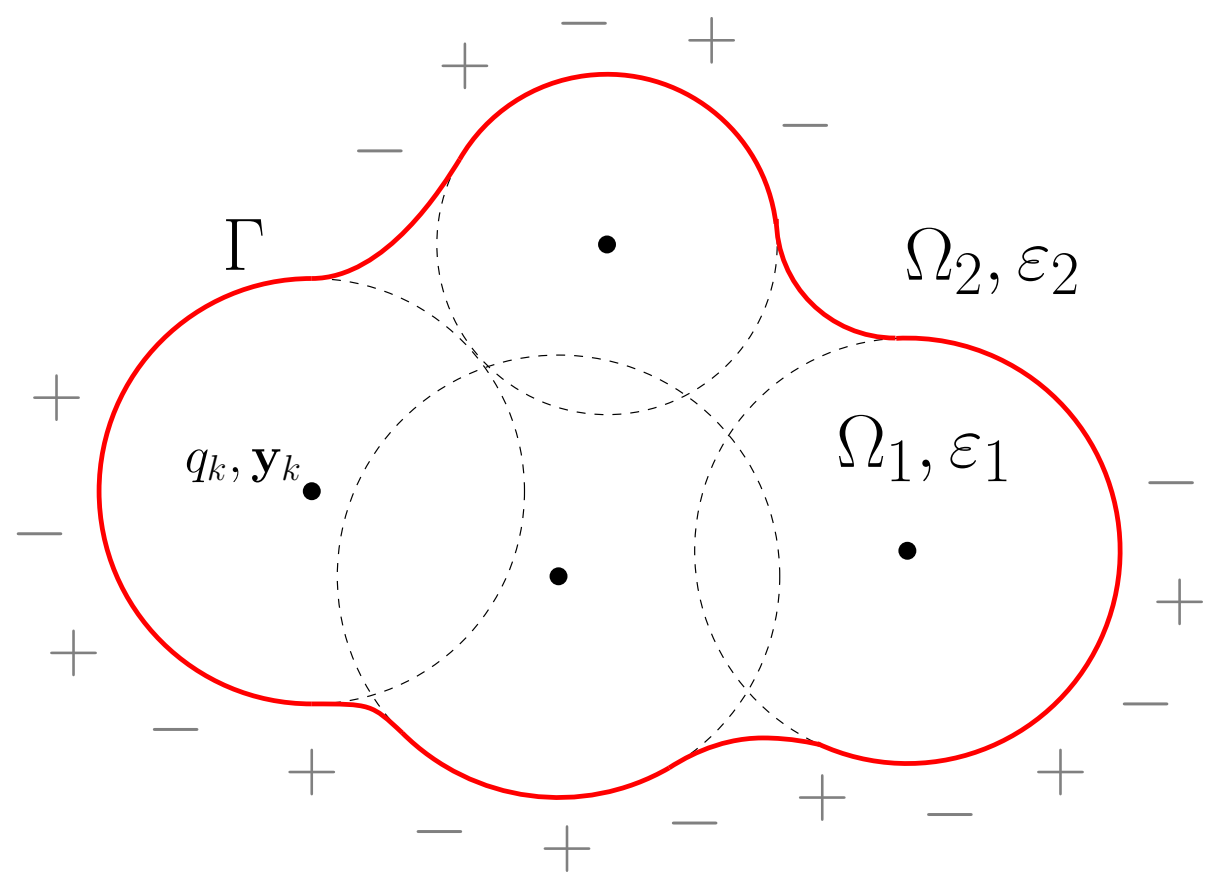

Figure 1: Poisson-Boltzmann implicit solvent model, solute domain $\Omega_{1}$ with dielectric constant $\varepsilon_{1}$, atomic charges $q_{k}$ located at $\mathbf{y}_{\mathbf{k}}, \mathrm{vdW}$ radii (dashed circles), solvent domain $\Omega_{2}$ with dielectric constant $\varepsilon_{2}$, dissolved salt ions $(+,-)$, dielectric interface $(\Gamma)$.

where the reaction field potential at an atomic position,

$$
\phi_{\text {reac }}\left(\mathbf{y}_{k}\right)=\lim _{\mathbf{x} \rightarrow \mathbf{y}_{k}}\left(\phi(\mathbf{x})-\sum_{j=1}^{N_{c}} \frac{q_{j}}{4 \pi\left|\mathbf{x}-\mathbf{y}_{j}\right|}\right),
$$

is the difference between the total potential and the Coulomb potential.

A variety of numerical methods have been applied to the PB model, including finitedifference $^{6-13}$, finite-element ${ }^{5[14[15}$, and boundary element $\frac{16}{20}$ methods. The present work is concerned with boundary element methods (BEM) which solve for the surface potential on a triangulation of the interface; these schemes benefit from rigorous enforcement of the interface conditions and the far-field boundary condition, but they face the difficulty of evaluating singular integrals and the expense of solving a dense linear system. The treecodeaccelerated boundary integral PB solver (TABI-PB ${ }^{19}$ ) addresses these issues using a simple collocation scheme to discretize the integrals and a treecode algorithm to reduce the cost of solving the linear system from $O\left(N^{2}\right)$ to $O(N \log N)$, where $N$ is the number of triangles representing the interface. 


\section{Dieletric Interface}

Several models have been used for the dielectric interface between the solute and solvent in implicit solvent simulations ${ }^{21 \mid 22}$. The van der Waals (vdW) surface, the simplest of these models, is the union of hard spheres with vdW radii representing the atoms comprising the biomolecule. The solvent accessible surface (SAS) is formed by tracing the center of a probe sphere representing a water molecule rolling along the exterior of the vdW surface; the SAS surface is equivalent to a vdW surface in which the vdW radii are increased by the probe sphere radius. The solvent excluded surface (SES) is formed by the inward facing surface of the probe sphere rolling along the vdW surface 23124 . The SES surface is comprised of spherical contact patches where the probe sphere touches the vdW surface, and toroidal reentrant patches formed by the inward facing surface of the probe sphere when it does not touch the vdW surface, i.e., when it is in contact with more than one solute atom. The skin surface $^{25} 27$ is comprised of spherical and hyperboloid patches constructed from a set of spheres through shrinking and convex combinations. The Gaussian surface ${ }^{28}$ is the level set of a linear combination of Gaussian functions centered at the solute atoms.

Several algorithms have been developed for triangulating these surfaces, where the input is the location and radii of the solute atoms and the output is a list of triangles that triangulate the surface. An alternative approach uses a level-set representation of the surface in an adaptive Cartesian grid ${ }^{[29 / 30}$. Publicly available surface triangulation codes include MSMS $^{31}$, EDTSurf ${ }^{32133}$, TMSmesh 2128 , and NanoShaper ${ }^{34}$. Previous work investigated the performance of SES surfaces and skin surfaces in the finite-difference Delphi code ${ }^{34}$, and the performance of Gaussian surfaces relative to SES surfaces in the boundary element fast multipole code AFMPB ${ }^{35}$. The present work focuses on the SES surface and compares the performance of MSMS and NanoShaper in computing the surface area and electrostatic solvation energy within the boundary element TABI-PB framework.

\section{MSMS}

MSMS, introduced by Sanner in 1995 31 , has gained widespread popularity for generating SES surface triangulations. After creating an analytical representation of the surface, the 
algorithm generates a triangulation of specified density by fitting predefined triangulated patches to the surface. The mesh resolution is controlled by the user-specified density parameter $d$ that sets the number of triangles in the triangulation of a given surface in units of vertices/angstrom ${ }^{2}$.

\section{NanoShaper}

NanoShaper, introduced by Decherchi and Rocchia in 2012 22 , implements the SES surface as well as several alternatives including the Gaussian and skin surfaces. In constructing an SES surface triangulation, NanoShaper first builds a description of the surface with a set of patches, analytically if possible or else with an approximation. The code then employs a ray-casting algorithm in which rays parallel to the coordinate axes are cast and intersections with the surface are calculated. The vertex positions of intersection are then used by the marching cubes algorithm to obtain the triangulation. The mesh resolution is controlled by the user-specified scaling parameter $s$ that sets the inverse side length of a cubic grid cell in units of angstroms.

The SES surface triangulations are commonly used to compute molecular properties such as the surface area, electrostatic potential, and electrostatic solvation energy of biomolecules. The present work investigates the triangulations produced by MSMS and NanoShaper in terms of their effect on the accuracy and efficiency of these computations.

\section{The TABI-PB Solver}

The TABI-PB solver ${ }^{19}$ relies on a reformulation of the PB Eq. (1) developed by Juffer et al. as a coupled set of boundary integral equations for the surface potential $\phi_{1}$ and its normal derivative $\partial \phi_{1} / \partial n$ on the dielectric interface ${ }^{17}$,

$$
\begin{aligned}
\frac{1}{2}(1+\varepsilon) \phi_{1}(\mathbf{x}) & =\int_{\Gamma}\left[K_{1}(\mathbf{x}, \mathbf{y}) \frac{\partial \phi_{1}(\mathbf{y})}{\partial n}+K_{2}(\mathbf{x}, \mathbf{y}) \phi_{1}(\mathbf{y})\right] d S_{\mathbf{y}}+S_{1}(\mathbf{x}), \quad \mathbf{x} \in \Gamma \\
\frac{1}{2}\left(1+\frac{1}{\varepsilon}\right) \frac{\partial \phi_{1}(\mathbf{x})}{\partial n} & =\int_{\Gamma}\left[K_{3}(\mathbf{x}, \mathbf{y}) \frac{\partial \phi_{1}(\mathbf{y})}{\partial n}+K_{4}(\mathbf{x}, \mathbf{y}) \phi_{1}(\mathbf{y})\right] d S_{\mathbf{y}}+S_{2}(\mathbf{x}), \quad \mathbf{x} \in \Gamma
\end{aligned}
$$


where $\varepsilon=\varepsilon_{1} / \varepsilon_{2}$ is the solute/solvent ratio of dielectric constants. The kernels $K_{1}, K_{2}, K_{3}, K_{4}$ depend on the Coulomb and screened Coulomb potentials,

$$
G_{0}(\mathbf{x}, \mathbf{y})=\frac{1}{4 \pi|\mathbf{x}-\mathbf{y}|}, \quad G_{\kappa}(\mathbf{x}, \mathbf{y})=\frac{e^{-\kappa|\mathbf{x}-\mathbf{y}|}}{4 \pi|\mathbf{x}-\mathbf{y}|}
$$

and the source terms are

$$
S_{1}(\mathbf{x})=\frac{1}{\varepsilon_{1}} \sum_{k=1}^{N_{c}} q_{k} G_{0}\left(\mathbf{x}, \mathbf{y}_{k}\right), \quad S_{2}(\mathbf{x})=\frac{1}{\varepsilon_{1}} \sum_{k=1}^{N_{c}} q_{k} \frac{\partial G_{0}\left(\mathbf{x}, \mathbf{y}_{k}\right)}{\partial n_{\mathbf{x}}}
$$

In this context the electrostatic solvation energy in Eq. (5) is obtained from the surface potential and its normal derivative,

$$
E_{\mathrm{sol}}=\frac{1}{2} \sum_{k=1}^{N_{c}} q_{k} \int_{\Gamma}\left[K_{1}\left(\mathbf{y}_{k}, \mathbf{y}\right) \frac{\partial \phi_{1}(\mathbf{y})}{\partial n}+K_{2}\left(\mathbf{y}_{k}, \mathbf{y}\right) \phi_{1}(\mathbf{y})\right] d S_{\mathbf{y}}
$$

The TABI-PB solver calculates the surface integrals using a boundary element method on the triangulated SES surface, where the collocation points are the triangle centroids. The resulting linear system for the surface potentials and their normal derivatives is solved by GMRES iteration, while a treecode algorithm is employed to accelerate the matrix-vector product in each step of the iteration ${ }^{19}$.

\section{METHODOLOGY}

To assess the SES surface triangulations produced by MSMS and NanoShaper, we compute the surface area $S_{\mathrm{a}}$ and electrostatic solvation energy $E_{\mathrm{sol}}$ for a test set of 38 biomolecules comprising peptides, proteins, and nucleic acid fragments, where $S_{\mathrm{a}}$ is computed by summing the areas of the triangles and $E_{\text {sol }}$ is computed using TABI-PB. In addition to examining the accuracy of these results, we report the total CPU time for the TABI-PB computation; the CPU time for generating the triangulation and other pre-processing steps is negligible in comparison to the boundary element computation time. Surface visualizations were generated with VTK ParaView.

The biomolecules in the test set, listed in Table 1, are those with widely available PDB

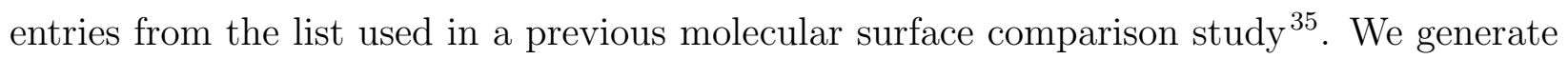


Table 1: PDB ID and number of atoms for test set of 38 biomolecules comprising proteins, peptides, and nucleic acid fragments $\underline{35}$.

\begin{tabular}{lllllllll}
\hline Index & 1 & 2 & 3 & 4 & 5 & 6 & 7 & 8 \\
PDB ID & $2 \mathrm{LWC}$ & $1 \mathrm{GNA}$ & $1 \mathrm{~S} 4 \mathrm{~J}$ & $1 \mathrm{CB} 3$ & $1 \mathrm{~V} 4 \mathrm{Z}$ & $1 \mathrm{BTQ}$ & $1 \mathrm{I} 2 \mathrm{X}$ & $1 \mathrm{AIE}$ \\
$\#$ atoms & 75 & 163 & 182 & 183 & 266 & 304 & 513 & 522 \\
\hline Index & 9 & 10 & 11 & 12 & 13 & 14 & 15 & 16 \\
PDB ID & $1 \mathrm{ZWF}$ & $375 \mathrm{D}$ & $440 \mathrm{D}$ & $4 \mathrm{HLI}$ & $3 \mathrm{ES} 0$ & $3 \mathrm{IM} 3$ & $2 \mathrm{IJI}$ & $1 \mathrm{COA}$ \\
\# atoms & 586 & 593 & 629 & 697 & 781 & 851 & 890 & 1057 \\
\hline Index & 17 & 18 & 19 & 20 & 21 & 22 & 23 & 24 \\
PDB ID & $2 \mathrm{AVP}$ & $1 \mathrm{SM} 5$ & $2 \mathrm{ONT}$ & $4 \mathrm{GSG}$ & $3 \mathrm{ICB}$ & $1 \mathrm{DCW}$ & $3 \mathrm{LDE}$ & $1 \mathrm{AYI}$ \\
$\#$ atoms & 1085 & 1137 & 1161 & 1195 & 1202 & 1257 & 1294 & 1365 \\
\hline Index & 25 & 26 & 27 & 28 & 29 & 30 & 31 & 32 \\
PDB ID & $2 \mathrm{YX} 5$ & $3 \mathrm{DFG}$ & $3 \mathrm{LOD}$ & $1 \mathrm{TR} 4$ & $1 \mathrm{RMP}$ & $1 \mathrm{IF} 4$ & $4 \mathrm{DUT}$ & $3 \mathrm{SQE}$ \\
$\#$ atoms & 1385 & 2198 & 2246 & 3423 & 3478 & 4071 & 4217 & 4647 \\
\hline Index & 33 & 34 & 35 & 36 & 37 & 38 & & \\
PDB ID & $1 \mathrm{HG} 8$ & $4 \mathrm{DPF}$ & $3 \mathrm{FR} 0$ & $2 \mathrm{H} 8 \mathrm{H}$ & $2 \mathrm{CEK}$ & $1 \mathrm{IL} 5$ & & \\
\# atoms & 4960 & 5824 & 6952 & 7084 & 8346 & 8349 & & \\
\hline
\end{tabular}

PQR files for each test biomolecule using PDB2PQR ${ }^{36}$ with the CHARMM force field and water molecules removed.

The MSMS triangulations were generated using density values $d=1,2,4,8,16$ and the NanoShaper triangulatons were generated using scaling parameter values $s=1,2,3,4,5$. For all surfaces a probe radius of $1.4 \AA$ was used.

The physical parameter values were ionic concentration $I_{s}=0.15 \mathrm{M}$, temperature $T=$ $300 \mathrm{~K}$, and solute and solvent dielectric constants $\varepsilon_{1}=1, \varepsilon_{2}=80$. The treecode parameters were multipole acceptance criterion $\theta=0.8$, Taylor series order $p=3$, and maximum number of particles in a leaf $N_{0}=500$. The GMRES tolerance was 1E-4, with 10 iterations between restarts and maximum number of iterations 110.

All computations were performed in serial on the University of Michigan FLUX cluster, 
with Intel Xeon CPUs running at either 2.5 or $2.8 \mathrm{GHz}$. In this system the exact processors could not be specified, so the timing results were averaged over multiple runs. The code was compiled with gfortran using the -O2 optimization flag. The newest version of the TABI-PB solver is available on GitHub at github.com/Treecodes/TABI-PB. The version of the TABI$\mathrm{PB}$ solver used in this work is available on GitHub at github.com/lwwilson/TABI-PB. We also note here that TABI-PB was recently implemented as an option in the APBS package developed at Pacific Northwest National Laboratory 37 .

\section{RESULTS AND DISCUSSION}

We first study geometric features of the surface triangulations by considering the triangle size, shape, and aspect ratio, and qualitatively comparing the generated surface meshes. We then extrapolate with respect to the number of triangles to calculate highly accurate converged values of the surface area and solvation energy; the converged result is the $y$ intercept of a simple extrapolation of the computed surface area or solvation energy versus $N^{-1}$ for the two highest resolution meshes produced by MSMS and NanoShaper. For some values of the density parameter $d$, MSMS produced spurious results for the larger molecules or failed to even produce a triangulation at all, as observed by previous investigators 29 ; in these cases the extrapolation used the highest resolution meshes for which MSMS did not fail.

\section{Triangulation Filter}

Both MSMS and NanoShaper produce a number of small or thin triangles which reduce the computational accuracy and efficiency, and it is common practice to delete them from the simulations. Hence in the present work, triangles are deleted if their area is less than $1 \mathrm{e}-5 \AA^{2}$ or if the distance between the centroids of two neighboring triangles is less than 1e-5 $\mathrm{A}$. Table 2 gives the percent of deleted triangles averaged over all triangulations using MSMS and NanoShaper. Among the deleted triangles, some had area less than machine precision and these are designated as zero-area triangles. With MSMS the deleted triangles 
are $0.064 \%$ of the total, while with NanoShaper the total is more than 100 times smaller. Table 2 also shows that most of the deleted MSMS triangles were zero-area, while none of this type were produced by NanoShaper.

Table 2: Triangulation filter results showing percent of deleted triangles and zero-area triangles, values shown are averaged over all triangulations using MSMS and NanoShaper, zero-area triangles are a subset of deleted triangles.

\begin{tabular}{ccc}
\hline code & deleted triangles & zero-area triangles \\
\hline MSMS & $6.4 \mathrm{e}-2 \%$ & $5.4 \mathrm{e}-2 \%$ \\
NanoShaper & $5.2 \mathrm{e}-4 \%$ & $0.0 \mathrm{e}-0 \%$ \\
\hline
\end{tabular}

\section{Triangle Aspect Ratios}

Even after filtering the triangulation as above, the aspect ratio of the remaining triangles can affect the computational performance. The aspect ratio of a triangular surface element is defined as the ratio of the longest to shortest sides. Figure 2 displays the (a) average aspect ratio, $r_{a v g}$, and (b) maximum aspect ratio, $r_{\text {max }}$, versus the number of triangles, $N$, for each triangulation, where $N$ varies from approximately $1 \mathrm{e}+3$ to $1 \mathrm{e}+6$, for the chosen density and scaling parameters. Figure $2 \mathrm{a}$ shows that the average aspect ratio of MSMS triangles is as large as $r_{a v g} \approx 30$ for small $N$ and decreases to approximately $r_{a v g} \approx 2$ for large $N$, while the average aspect ratio of NanoShaper triangles is closer to $r_{a v g} \approx 1$ for all $N$. Figure $2 \mathrm{~b}$ shows that the maximum aspect ratio of MSMS triangles varies between approximately $1 \mathrm{e}+2$ and $2 \mathrm{e}+3$, while the maximum aspect ratio of NanoShaper triangles is below $1 \mathrm{e}+1$ across all triangulations.

\section{Surface Mesh Features}

Figure 3 displays the triangulation and surface potential for a representative protein (1AIE) using (a) MSMS and (b) NanoShaper with similar resolution, $N \approx 3 \mathrm{e}+4$ triangles in each 


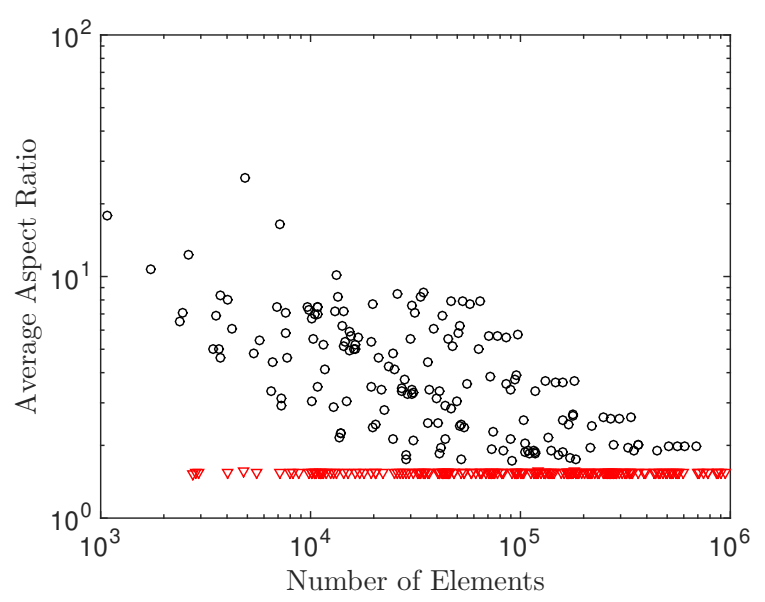

(a) average aspect ratio, $r_{\text {avg }}$

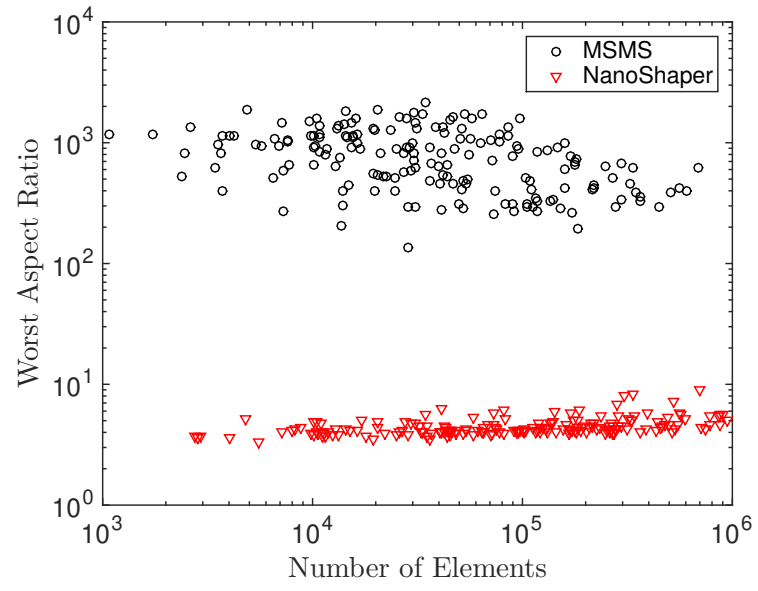

(b) maximum aspect ratio, $r_{\max }$

Figure 2: Triangle aspect ratio versus number of elements $N$ for each generated surface, (a) average aspect ratio, $r_{\text {avg }}$, (b) maximum aspect ratio, $r_{\text {max }}$, MSMS (o, black), NanoShaper $(\nabla$, red $)$.

case. The surfaces are similar at first glance, although the NanoShaper surface appears slightly smoother than the MSMS surface. Figure 4 displays a zoom of the triangulations, where several irregular features are highlighted; in the MSMS mesh, green boxes enclose stitches formed by high aspect ratio triangles, and a white box encloses a cusp formed by neighboring triangles that meet at a acute angle, while in the NanoShaper mesh, a yellow box encloses a possible irregular feature, which could in fact simply be an artifact of the surface lighting. It should be noted that irregular features are present in the MSMS mesh even after filtering; by contrast, the NanoShaper mesh is relatively free of such irregular features. The irregular features diminish the efficiency of the calculations; as shown below, calculations using MSMS meshes require more iterations to converge in comparison with calculations using NanoShaper meshes.

\section{Dependence of $S_{a}$ and $E_{\mathrm{sol}}$ on Mesh Resolution}

In this section we examine the dependence of the surface area $S_{a}$ and solvation energy $E_{\text {sol }}$ on the mesh resolution for four representative proteins (1AIE, 1HG8, 3FR0, 1IL5). Figure 5 plots $S_{a}$ and Fig. 6 plots $E_{\text {sol }}$ versus $N^{-1}$, where $N$ is the number of surface elements. As 


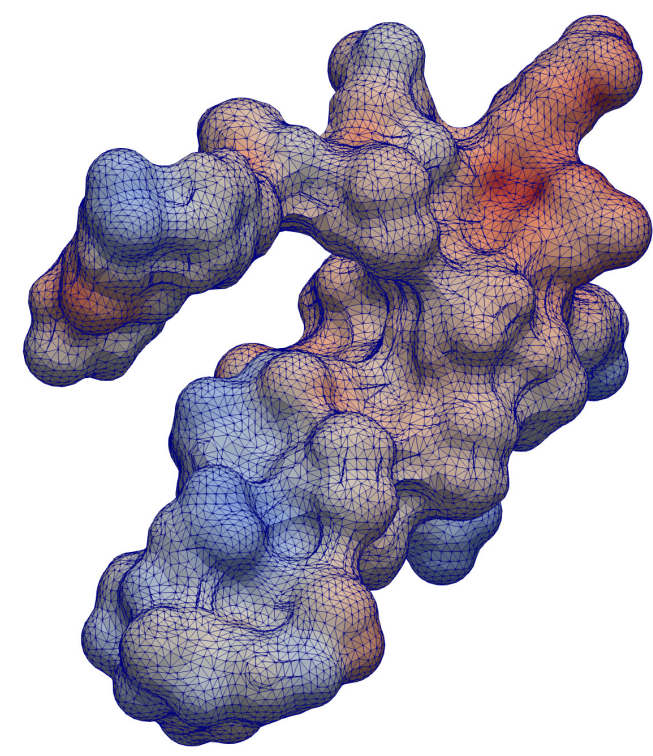

(a) MSMS
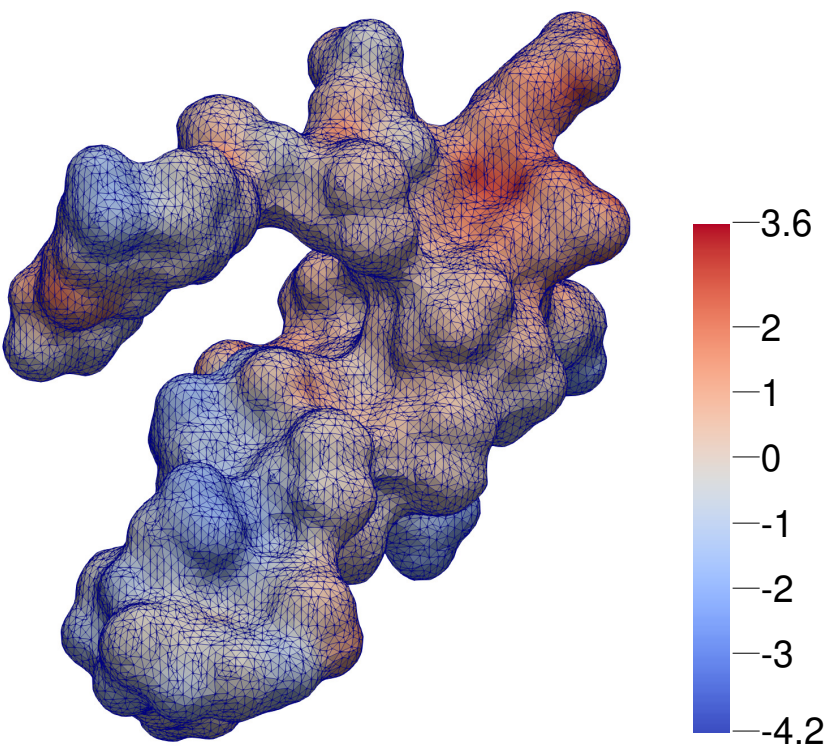

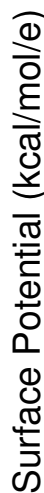

Figure 3: Protein 1AIE, SES triangulation and electrostatic potential, (a) MSMS, density $d=6, N=31480$ triangles, (b) NanoShaper, scaling parameter $s=2, N=32208$ triangles.

expected the MSMS and NanoShaper results for $S_{a}$ and $E_{\text {sol }}$ converge to similar limits since both codes approximate the solvent excluded surface, but several differences can be seen in their dependence on $N$.

First, concerning the surface area in Fig. 5, the MSMS and NanoShaper results converge to almost the same values in Fig. 5a,d (1AIE, 1IL5), but in Fig. 5b,c (1HG8, 3FR0), the NanoShaper surface area is 2-3\% larger than the MSMS surface area. In all cases the convergence with $N^{-1}$ is smooth. The MSMS results approach their limit somewhat faster, although MSMS was unable to generate reliable meshes with larger values of $N$; either it fails to produce a mesh, or the generated mesh was poorly formed. The largest value we obtained using MSMS was $N \approx 2 \mathrm{e}+6$, whereas NanoShaper had no such limitation. Hence if it is necessary to generate a very dense mesh, or even a less dense mesh for a biomolecule with a large surface area, then NanoShaper has an advantage.

Second, concerning the solvation energy in Fig. 6, the MSMS and NanoShaper results converge to almost the same value. The MSMS results again approach their limiting value 


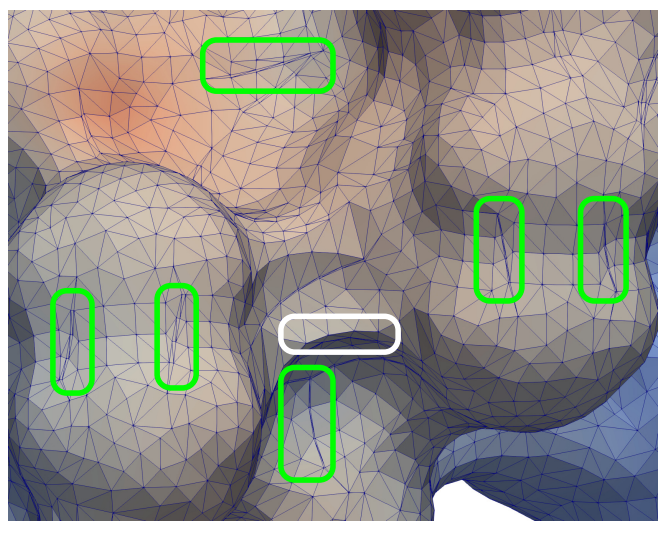

(a) MSMS, zoomed

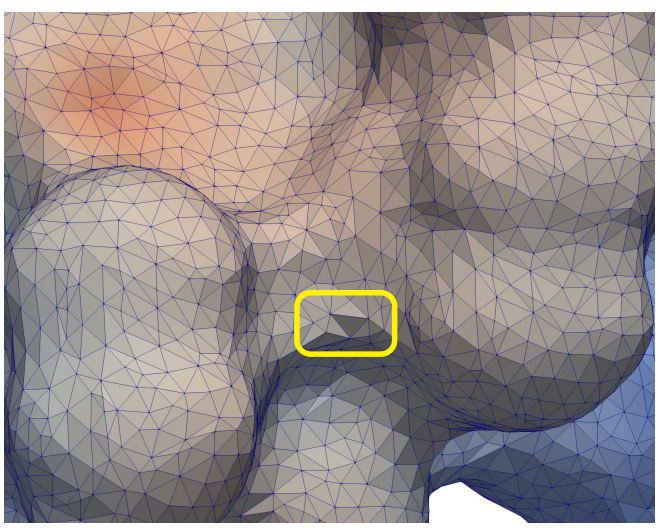

(b) NanoShaper, zoomed

Figure 4: Protein 1AIE, zoom of SES triangulation, (a) MSMS, density $d=6, N=$ 31480 triangles, green boxes enclose stitches formed by high aspect ratio triangles, white box encloses a cusp formed by neighboring triangles that meet at an acute angle, (b) NanoShaper, scaling parameter $s=2, N=32208$ triangles, yellow box encloses a possible irregular feature.

somewhat faster than the NanoShaper results, although the NanoShaper dependence on $N$ is smoother than the MSMS dependence.

Figure 7 displays the (a) surface area $S_{a}$ and (b) solvation energy $E_{\text {sol }}$ for the entire set of 38 biomolecules, where the NanoShaper results are plotted versus MSMS results. In this case to reduce the dependence of the computed values on the mesh resolution $N$, we extrapolated the computed $S_{a}$ and $E_{\text {sol }}$ to the limit $N \rightarrow \infty$ using the two highest resolution meshes, density $d=8,16$ for MSMS and scaling factor $s=4,5$ for NanoShaper. The correspondence between MSMS and NanoShaper results in Fig. 7 is very good, except for two molecules, 1I2X and 375D, which consist of multiple domains, for which MSMS did not generate an accurate mesh. These two anomalous cases are indicated by the two markers furthest away from the diagonal line in Figs. 7a,b. In addition, MSMS failed to produce surfaces for 13 other runs, and produced highly distorted surfaces with spurious solvation energy for 3 more runs. These 16 spurious runs were removed from the calculations in this section. By contrast, NanoShaper failed in only one case, a low resolution mesh with scaling factor $s=1$ for the smallest molecule in the test set (2LWC, 75 atoms). 

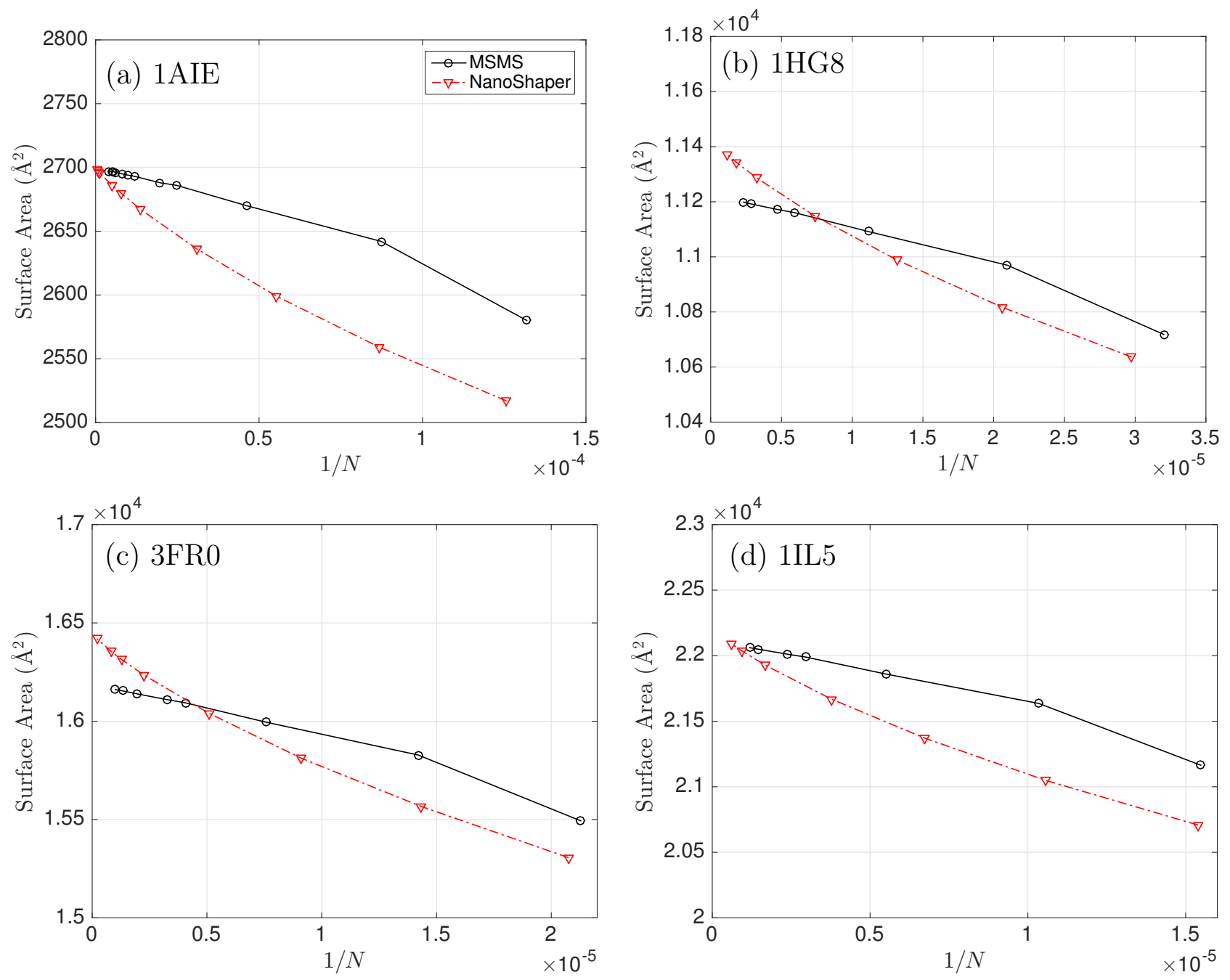

Figure 5: $\quad$ Surface area $S_{a}$ versus $N^{-1}$ for four representative proteins, where $N$ is the number of triangles, MSMS (black, solid line, o), NanoShaper (red, dashed line, $\nabla$ ).

\section{Computational Efficiency}

Figure 8 a displays the total TABI-PB run time versus the number of triangles $N$ for computing the solvation energy $E_{\text {sol }}$ using MSMS and NanoShaper meshes, where the solid lines are least squares fits to the data. The run time for creating and filtering the meshes is less than eight seconds in all cases, and thus constitutes a negligible fraction of the total run time. The results show that in general, NanoShaper meshes require less run time than MSMS meshes. This is supported by Fig. 8b showing the number of GMRES iterations in each case, where the maximum number of iterations was set to 110. The results show that in general, 

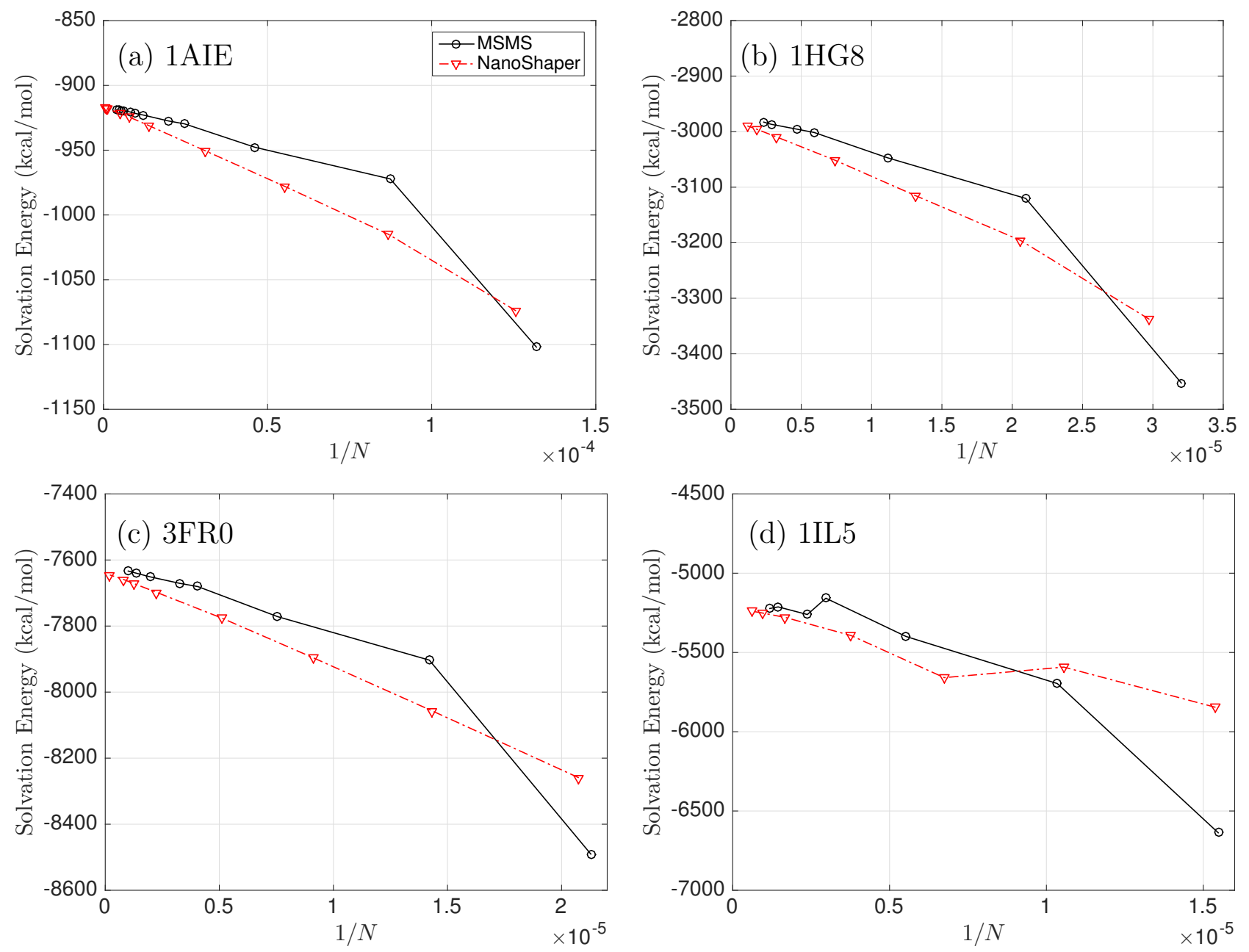

Figure 6: Solvation energy $E_{\text {sol }}$ versus $N^{-1}$ for four representative proteins, where $N$ is the number of triangles, MSMS (black, solid line, o), NanoShaper (red, dashed line, $\nabla$ ).

NanoShaper meshes require fewer GMRES iterations than MSMS meshes. Moreover, in the case of MSMS, the iteration limit was reached in 23 out 177 meshes, while in the case of NanoShaper, the iteration limit was never reached.

Table 3 displays the average run time and average number of GMRES iterations per triangle for each mesh type over the entire set of 38 biomolecules. The results show that NanoShaper meshes require about $2 / 3$ of the run time and $1 / 4$ of the number of iterations required by MSMS meshes. The larger number of GMRES iterations required for MSMS meshes is attributed to the presence of triangles with large aspect ratio, as seen in Fig. 2 , and irregular features in the generated surfaces, as seen in Fig. 4 . 


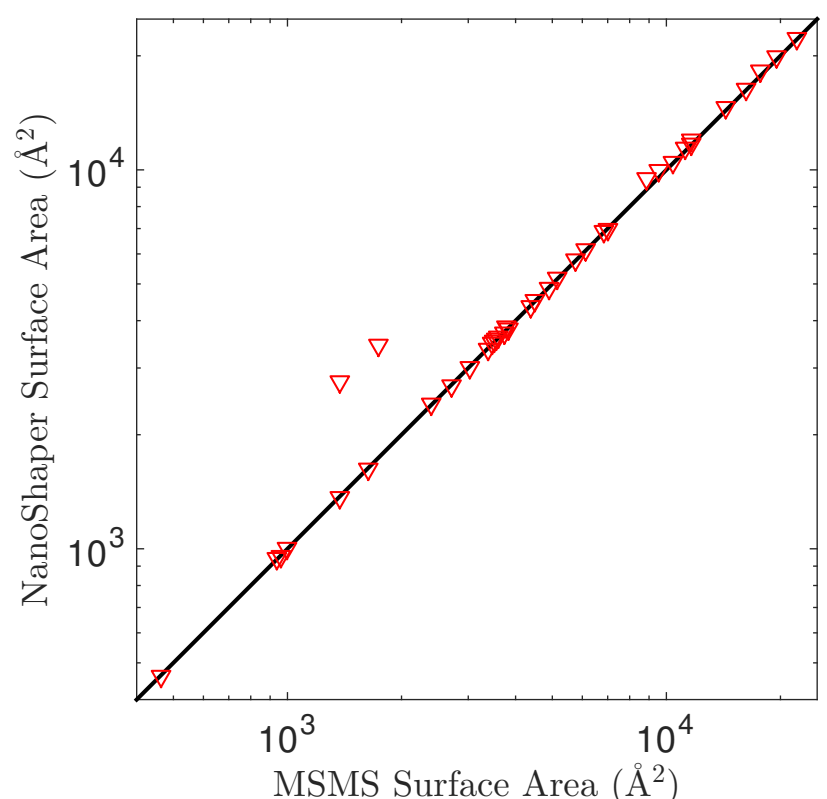

(a)

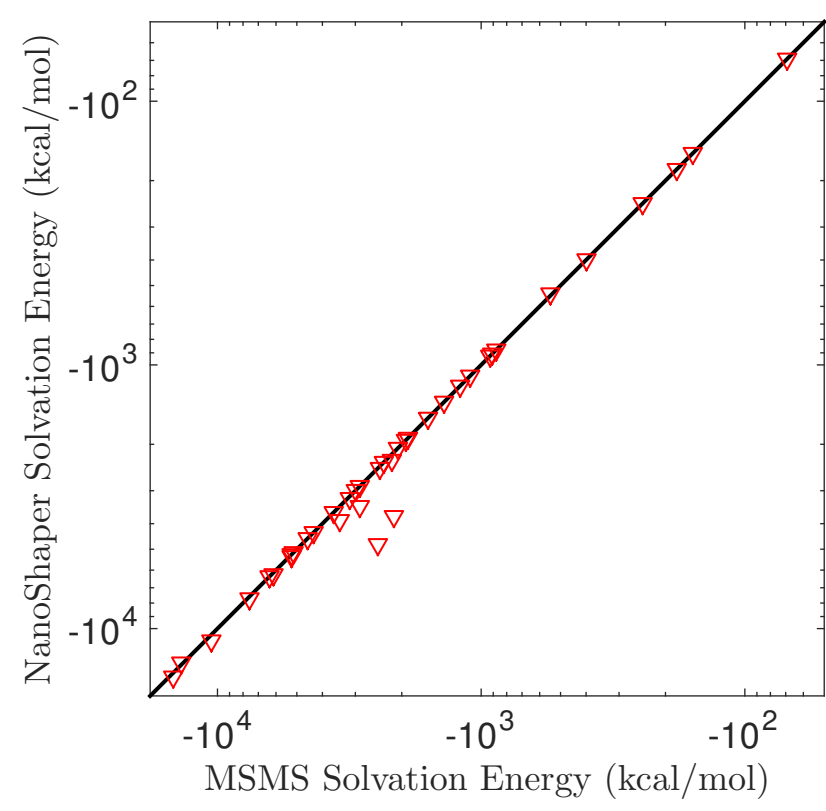

(b)

Figure 7: NanoShaper versus MSMS results for entire set of 38 biomolecules using values extrapolated to the limit $N \rightarrow \infty$, (a) surface area $S_{a}$, (b) solvation energy $E_{\text {sol }}$, black lines indicate perfect correspondence.

\section{CONCLUSIONS}

We compared the performance of MSMS and NanoShaper, two widely used codes for triangulating the solvent excluded surface (SES) in Poisson-Boltzmann simulations of solvated biomolecules. Comparisons were made of the surface area and electrostatic solvation energy, where the latter calculations were performed using the treecode-accelerated boundary integral (TABI-PB) solver which utilizes a well-conditioned boundary integral formulation and

Table 3: Average run time (s) and average number of GMRES iterations per triangle for MSMS and NanoShaper meshes over entire set of 38 biomolecules.

\begin{tabular}{c|c|c}
\hline & average run time $(\mathrm{s}) /$ triangle & average iterations/triangle \\
\hline MSMS & $6.67 \mathrm{e}-3$ & $1.17 \mathrm{e}-3$ \\
NanoShaper & $4.19 \mathrm{e}-3$ & $2.92 \mathrm{e}-4$ \\
\hline
\end{tabular}




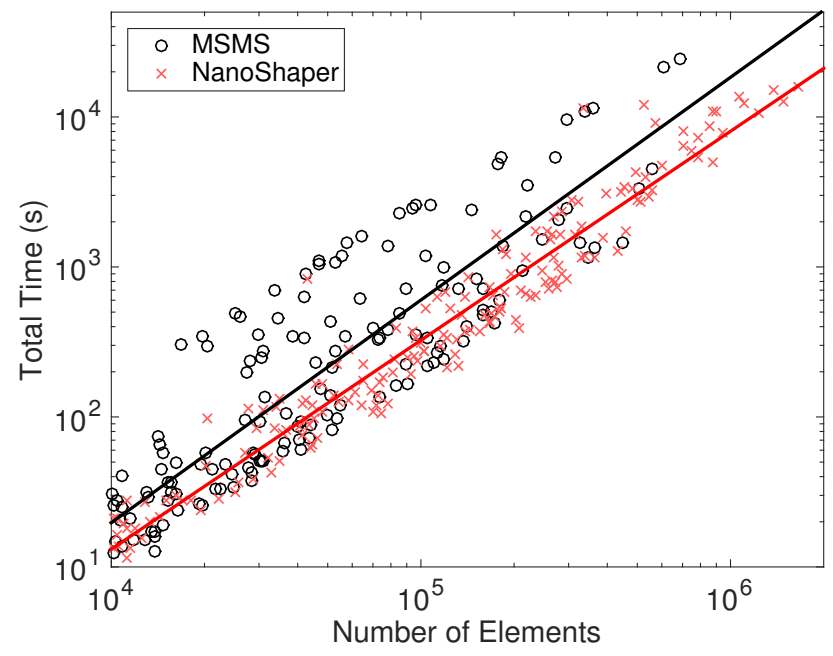

(a)

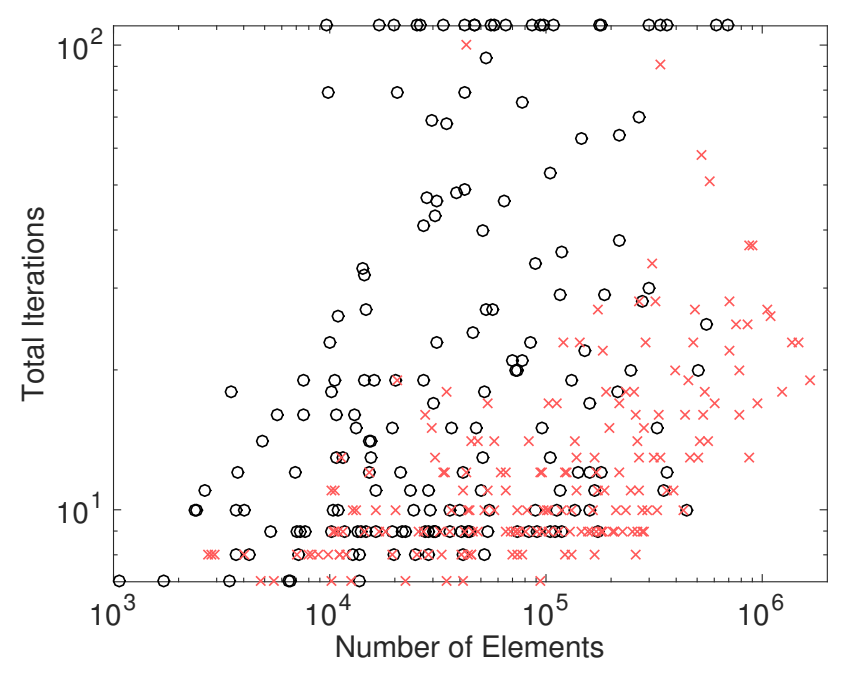

(b)

Figure 8: Computational efficiency, total run time of TABI-PB for computing solvation energy $E_{\text {sol }}$ using MSMS (o, black) and NanoShaper (×, red) versus number of triangles $N$, (a) run time (s), solid lines are least squares fits, (b) number of GMRES iterations (maximum 110).

centroid collocation on the SES triangulation. In these calculations, the linear system for the electrostatic potential and its normal derivative on the SES is solved by GMRES iteration. The matrix-vector product in each step of GMRES is computed by a treecode which reduces the computational cost from $O\left(N^{2}\right)$ to $O(N \log N)$, where $N$ is the number of elements in the surface triangulation.

The MSMS and NanoShaper codes were compared for a test set of 38 biomolecules. The meshes produced by the two codes are qualitatively similar, although the MSMS meshes often contained triangles of exceedingly small area and high aspect ratio. The computed values of the surface area and solvation energy produced by MSMS and NanoShaper meshes often agree to within several percent. NanoShaper meshes were more computationally efficient, requiring less run time and fewer GMRES iterations than MSMS meshes. Furthermore, NanoShaper was consistently able to produce higher resolution meshes than MSMS, and NanoShaper solvation energies exhibited smoother convergence with increasing mesh resolution. A version of TABI-PB using NanoShaper was recently implemented as an option in $\mathrm{APBS}$. 37 . 


\section{ACKNOWLEDGMENTS}

This work was supported by NSF grant DMS-1819094 and a catalyst grant from the Michigan Institute for Computational Discovery and Engineering (MICDE). Leighton Wilson was supported by the Department of Defense (DoD) through the National Defense Science \& Engineering Graduate Fellowship (NDSEG) Program. 


\section{References}

1. B. Roux and T. Simonson, Biophys. Chem. 78, 1 (1999).

2. Z. Zhang, S. Witham, and E. Alexov, Phys. Biol. 8, 035001 (2011).

3. J. Tomasi, Theor. Chem. Acc. 112, 184 (2004).

4. N. A. Baker, in Numerical Computer Methods, Part D, edited by L. Brand and M. L. Johnson (Academic Press, 2004), vol. 383 of Methods in Enzymology, chap. 5, pp. 94-118, 1 st ed.

5. B. Lu, Y. C. Zhou, M. J. Holst, and J. A. McCammon, Commun. Comput. Phys. 3, 973 (2008).

6. M. J. Holst and F. Saied, J. Comput. Chem. 16, 337 (1995).

7. N. A. Baker, D. Sept, S. Joseph, M. J. Holst, and J. A. McCammon, Proc. Natl. Acad. Sci. U.S.A. 98, 10037 (2001).

8. R. Luo, L. David, and M. K. Gilson, J. Comput. Chem. 23, 1244 (2002).

9. J. Wang and R. Luo, J. Comput. Chem. 31, 1689 (2010).

10. D. Chen, Z. Chen, C. Chen, W. Geng, and G. W. Wei, J. Comput. Chem. 32, 756 (2011).

11. A. H. Boschitsch and M. O. Fenley, J. Chem. Theory Comput. 7, 1524 (2011).

12. W. Geng and S. Zhao, Mol. Based Math. Biol. 1, 109 (2012).

13. L. Wilson and S. Zhao, Int. J. Numer. Anal. Model. 13, 852 (2016).

14. M. J. Holst, N. A. Baker, and F. Wang, J. Comput. Chem. 21, 1319 (2000).

15. N. A. Baker, M. J. Holst, and F. Wang, J. Comput. Chem. 21, 1343 (2000).

16. J. Liang and S. Subramaniam, Biophys. J. 73, 1830 (1997). 
17. A. H. Juffer, E. F. F. Botta, B. A. M. van Keulen, A. van der Ploeg, and H. J. C. Berendsen, J. Comput. Phys. 97, 144 (1991).

18. A. H. Boschitsch, M. O. Fenley, and H.-X. Zhou, J. Phys. Chem. B 106, 2741 (2002).

19. W. Geng and R. Krasny, J. Comput. Phys. 247, 62 (2013).

20. C. D. Cooper, J. P. Bardhan, and L. A. Barba, Comput. Phys. Commun. 185, 720 (2014).

21. M. Chen and B. Lu, J. Chem. Theory Comput. 7, 203 (2011).

22. S. Decherchi and W. Rocchia, PLoS ONE 8, e59744 (2013).

23. F. M. Richards, Annu. Rev. Biophys. Bioeng. 6, 151 (1977).

24. M. L. Connolly, J. Appl. Crystallogr. 18, 499 (1985).

25. W. Rocchia, S. Sridharan, A. Nicholls, E. G. Alexov, A. Chiabrera, and B. Honig, J. Comput. Chem. 23, 128 (2002).

26. W. Rocchia, Math. Comput. Model. 41, 1109 (2005).

27. H.-L. Cheng and X. Shi, Comput. Geom. 42, 192 (2009).

28. M. Chen, B. Tu, and B. Lu, J. Mol. Graph. Model. 38, 411 (2012).

29. T. Can, C.-I. Chen, and Y.-F. Wang, J. Mol. Graph. Model. 25, 442- (2006).

30. R. Egan and F. Gibou, J. Comput. Phys. 374, 91 (2018).

31. M. F. Sanner, A. J. Olson, and J.-C. Spehner, in Proceedings of the 11th ACM Symposium on Comput. Geom. (Association for Computing Machinery, 1995), pp. C6-C7.

32. D. Xu and Y. Zhang, PLoS ONE 4, e8140 (2009).

33. D. Xu, H. Li, and Y. Zhang, J. Comput. Biol. 20, 805 (2013).

34. S. Decherchi, J. Colmenares, C. E. Catalano, M. Spagnuolo, E. Alexov, and W. Rocchia, Commun. Comput. Phys. 13, 61 (2013). 
35. T. Liu, M. Chen, and B. Lu, J. Mol. Model. 21, 113 (2015).

36. T. J. Dolinsky, J. E. Nielsen, J. A. McCammon, and N. A. Baker, Nucleic Acids Res. 32, W665 (2004).

37. E. Jurrus, D. Engel, K. Star, K. Monson, J. Brandi, L. E. Felberg, D. H. Brookes, L. Wilson, J. Chen, K. Liles, et al., Protein Sci. 27, 112 (2017). 